\title{
Detection of rotavirus by a latex agglutination test, Rotalex: comparison with electron microscopy, immunofluorescence, polyacrylamide gel electrophoresis, and enzyme linked immunosorbent
} assay

\author{
RB MOOSAI, R ALCOCK, TM BELL, FR LAIDLER, JSM PEIRIS, ${ }^{*}$ AP WYN-JONES, $\dagger$ \\ CR MADELEY
}

From the Department of Virology, Royal Victoria Infirmary and University of Newcastle upon Tyne, Newcastle upon Tyne, and the †Department of Biology, Sunderland Polytechnic, Sunderland, Tyne and Wear

SUMmARY A commercially available latex agglutination test, Rotalex (Orion Diagnostics, Finland), for detecting rotaviruses was evaluated in comparison with four other tests (electron microscopy, immunofluorescence, polyacrylamide gel electrophoresis, and enzyme linked immunosorbent assay) routinely used in our laboratories. Although Rotalex was the least complex method, it showed lack of specificity and sensitivity when carried out according to the manufacturer's instructions. Four basic modifications of Rotalex are described. These include the use of Hank's balanced salt solution, increasing the incubation time to $20 \mathrm{~min}$, reading the agglutination result by an experienced observer, and the use of $50 \mathrm{~mm}$ square glass plates. The modified procedure gave results which were comparable with those obtained by electron microscopy, immunofluorescence, polyacrylamide gel electrophoresis, and enzyme linked immunosorbent assay. The latter techniques, when used to detect rotavirus, all gave similar results.

Rotaviruses are among the commonest viruses and are readily detectable in stools by a variety of methods. These range from antibody based tests such as immunofluorescence, ${ }^{12}$ enzyme linked immunosorbent assay (ELISA), ${ }^{34}$ radioimmunoassay, ${ }^{56}$ and counterimmunoelectro-osmophoresis ${ }^{78}$ to methods which do not require the use of antibody such as electron microscopy ${ }^{10}$ and polyacrylamide gel electrophoresis (PAGE) of viral ribonucleic acid. ${ }^{1-13}$ Recently, a latex agglutination test, Rotalex (Orion Diagnostics, Helsinki, Finland), has been developed for the detection of rotavirus. The test depends on the agglutination of latex particles coated with specific antibody by rotavirus present in stool extracts. The main advantages claimed for this test are that it can be carried out on a slide, read with the naked eye or a hand lens, and is suitable for use on the ward in paediatric hospitals. The availa-

Accepted for publication 4 February 1985

${ }^{*}$ Present address: Department of Microbiology, University of Peradeniya, Peradeniya, Sri Lanka. bility of Rotalex prompted us to compare it with the other tests routinely used in our laboratories: immunofluorescence, PAGE, electron microscopy, and ELISA under double blind conditions. Each test was compared with the others, and this paper reports the results.

\section{Material and methods}

SPECIMENS

All stool specimens used in this study had been stored at $-70^{\circ} \mathrm{C}$ for varying times before use. They came from children and infants admitted to hospitals in the north east of England and had originally been submitted to the routine diagnostic laboratory; they had been kept at $4^{\circ} \mathrm{C}$ for a maximum of $48 \mathrm{~h}$ before freezing.

The stools used in this study fell into three categories: (a) positive for rotavirus by electron microscopy; (b) negative for rotavirus by electron microscopy; and (c) large volume samples negative for rotavirus by more than one method, which were 
used as a diluent to produce a series of specimens containing progressively fewer rotaviruses but with a constant amount of faecal material in each. These stools were also shown, by an ELISA technique, to be free of antibody to the Wa strain of rotavirus. The first two categories were used as test specimens and were diluted in Rotalex buffer or Hank's balanced salt solution (HBSS) as described in the Results section.

\section{IMMUNOFLUORESCENCE}

The indirect immunofluorescence method described by Moosai $e t \mathrm{al}^{2}$ was used to detect human rotavirus in the samples. Extracts were inoculated on to coverslip cultures of LLC-MK2 cells in the presence of crystalline trypsin $(20 \mu \mathrm{g} / \mathrm{ml})$ and tested by immunofluorescence $24 \mathrm{~h}$ later. The antiserum used was raised in a rabbit using an extract of a single human rotavirus positive stool, purified by isopycnic gradient centrifugation. The rabbit was previously negative for rotavirus antibody, as determined by immunofluorescence. Before use it was shown to be specific and devoid of non-specificity by the criteria proposed by Gardner and McQuillin. ${ }^{14}$ The conjugate used was a fluorescein isothiocyanate-labelled sheep antirabbit globulin (Wellcome Diagnostics, Dartford, Kent).

\section{ELECTRON MICROSCOPY}

Specimens for electron microscopy were extracted in $5 \mathrm{ml}$ of HBSS, shaken by hand, and clarified in an MSE Super Minor centrifuge at $3000 \mathrm{rpm}$ for 10 min at $4^{\circ} \mathrm{C}$. The supernatant was then centrifuged at $40000 \mathrm{rpm}$ for $1 \mathrm{~h}$ at $4^{\circ} \mathrm{C}$ in a Beckman L2-65B centrifuge using a SW55 swing out rotor to sediment any virus. The pellet was resuspended in six drops of $0.1 \%$ bacitracin in distilled water and examined by negative contrast in a Philips EM 300 electron microscope using $2 \%$ phosphotungstic acid adjusted to $\mathrm{pH} 7$ with $0 \cdot 1 \mathrm{M}$ potassium hydroxide.

\section{POLYACR YLAMIDE GEL ELECTROPHORESIS}

Aliquots of unpurified suspensions were treated with an equal volume of $10 \%$ sodium dodecyl sulphate in distilled water and incubated at $37^{\circ} \mathrm{C}$ for $30 \mathrm{~min}$ to release viral nucleic acid. The treated extract was then mixed 1:1 with sample buffer $(62$ $\mathrm{mM}$ Tris-HC1, $\mathrm{pH} 6.8$, containing $3 \%$ sodium dodecyl sulphate, $5 \%$ 2-mercaptoethanol, and $40 \%$ glycerol) for electrophoresis.

Electrophoresis was conducted overnight (about $16 \mathrm{~h}$ ) at room temperature in $1.5 \mathrm{~mm}$ thick, $7.5 \%$ polyacrylamide slab gels with a $3 \%$ stacking gel, using the discontinuous buffer system described by Laemmli. ${ }^{15}$

Gels were stained with silver nitrate using the method of Herring et al $^{12}$ with minor modifications. The staining time in silver nitrate was reduced to $1 \mathrm{~h}$ and the gels were stained in suspension after removal from the supporting glass plates. The solutions were used without prior degassing and the stained gels were photographed by transmitted light over an $x$ ray viewing box using $35 \mathrm{~mm}$ Ilford Pan F film and a $55 \mathrm{~mm}$ Micro-Nikkor lens.

\section{ENZYME LINKED IMMUNOSORBENT ASSAY}

The ELISA technique was performed using a monoclonal antihuman rotavirus ascitic fluid as capture antibody. This reagent was prepared by one of us (JSMP) in Dr TH Flewett's laboratory in Birmingham and reacts with the internal group antigen of the rotavirus particle. ${ }^{16}$ Briefly, Dynatech B plates (Dynatech, Sussex) were coated with the monoclonal antibody $(100 \mu \mathrm{l}$ per well) at a dilution of $10^{-4}$ in carbonate-bicarbonate buffer, $\mathrm{pH} \mathrm{9.6.} \mathrm{The}$ antibody was allowed to adsorb overnight at $4^{\circ} \mathrm{C}$. Monoclonal antibody ascitic fluid, prepared against an irrelevant antigen (West Nile virus), was used at the same dilution as a negative control capture antibody to monitor non-specificity. Samples $(50 \mu \mathrm{l})$ were added and allowed to react for $3 \mathrm{~h}$ at $37^{\circ} \mathrm{C}$, after which indicator antibody $(50 \mu \mathrm{l})$ was applied under the same conditions. The indicator antibody was the same monoclonal reagent used earlier, tagged with alkaline phosphatase (Sigma Chemical Co). One hundred microlitres of p-nitro-phenyl phosphate substrate was then added and after 60 min incubation at $37^{\circ} \mathrm{C}$ the reaction was stopped with $3 \mathrm{M}$ sodium hydroxide $(30 \mu \mathrm{l}$ per well). The plates were washed at each stage of the process by immersion six times in two changes of a solution of Tween 80 and saline, and after reaction with substrate they were read either by eye or in an ELISA reader at $410 \mathrm{~nm}$. A sample was considered to be positive for rotavirus if it had an optical density that was more than twice that obtained with the negative control.

\section{ROTALEX}

Rotalex kits were obtained from Northumbria Biologicals (Cramlington, England) and the test was initially performed exactly according to the instructions enclosed with the kits. Briefly, this entailed preparing a $10 \%$ stool suspension in Rotalex buffer (provided by the manufacturer, composition not available), allowing the sample to stand for $30 \mathrm{~min}$, centrifuging at $1200 \mathrm{~g}$ for $20 \mathrm{~min}$, and then transferring two drops of the supernatant to a test slide.

One drop was mixed with test latex (coated with antibody to rotavirus), left for $2 \mathrm{~min}$, and examined for the development of agglutination. Control latex was added to the second drop and this served as the 
negative control. A positive virus control which showed agglutination with the test latex was provided with the kit and was included in all experiments.

The above method was modified during the course of our investigation (see below). In some experiments stools were diluted initially in HBSS and then in Rotalex buffer before testing, and the agglutination reaction was read after 2,10 , and 20 min.

\section{Results}

Preliminary experiments on Rotalex alone were done to get the "feel" of the test and to discover any practical problems in its use. A plastic surface was found to be unsuitable because the latex bound to it non-specifically. This was subsequently confirmed by the manufacturers. The latex was provided in dropping bottles and the drops produced were too large for standard $3^{\prime \prime} \times 1^{\prime \prime}(75 \mathrm{~mm} \times 25 \mathrm{~mm})$ microscope slides. Subsequent tests were therefore done on larger glass plates, 2 inch square $(50 \mathrm{~mm} \times$ $50 \mathrm{~mm}$ ). The length of incubation of the reaction mixture before reading was also important.

A series of experiments was designed to evaluate the suitability of Rotalex for detecting rotavirus in clinical specimens in a comparative study with the four other tests used in our laboratories for detecting rotavirus: PAGE, immunofluorescence, electron microscopy, and ELISA. Eighteen stool samples (nine of which were previously positive for rotavirus by electron microscopy) were examined at dilutions of $1 / 10$ and $1 / 100$. The $1 / 10$ dilutions were made in Rotalex buffer (for latex agglutination) or HBSS (for the other tests). The 1/100 dilutions were prepared by diluting the 18 samples $1 / 10$ in HBSS and then a further $1 / 10$ in either Rotalex buffer (for latex agglutination) or HBSS (for the other tests). Before being used as second stage diluents, the Rotalex buffer and HBSS were mixed with a stool negative for rotavirus to give $10 \%$ suspensions and hence the same amount of particulate faecal material in each of the test specimens.

Aliquots of each of the two dilutions (1/10 and $1 / 100$ ) of the 18 samples were dispensed for each of the five tests. This gave 180 aliquots for testing. One of us (TMB) then allocated a random number, between 1 and 180 , to each aliquot. He then separated sets of specimens for the various tests, placed them in numerical order, and gave them to other members of the team for testing blind.

Table 1 shows the results obtained with the Rotalex test system read after 2,10 , and 20 min by three different observers $(\mathrm{A}, \mathrm{B}$, and $\mathrm{C})$. With the positive specimens; the proportion read by all three observers as positive was least at $2 \mathrm{~min}$, had increased by $10 \mathrm{~min}$, and was higher still after $20 \mathrm{~min}$. This extended incubation time also raised the number of negative results read incorrectly as positive. This was particularly pronounced with the stools diluted $1 / 10$ in Rotalex buffer. Diluting stools initially $1 / 10$ in HBSS and then to a final dilution of 1/100 with Rotalex buffer largely abolished these false positive results. Those remaining were recorded only by the least experienced observer, $C$, who had not read any of the results undertaken with Rotalex in preliminary experiments.

Table 2 shows the results of the Rotalex test read after $20 \mathrm{~min}$ compared with PAGE, ELISA, immunofluorescence, and electron microscopy. In contrast to Rotalex, which missed several of the positive samples and falsely scored some negative results as positive with the $1 / 10$ dilutions, rotavirus was detected at both dilutions in all the positive samples by PAGE, ELISA, immunofluorescence, and electron microscopy, and no false positive results were found.

This dilution sequence (HBSS and then Rotalex buffer) was therefore followed when preparing samples for latex agglutination in a second experiment designed to assess the sensitivity of Rotalex relative to electron microscopy, PAGE, ELISA, and immunofluorescence. Five stool samples (three of which were positive for rotavirus by electron microscopy) were submitted for coding. Four were randomly selected (blind) and $10 \%$ suspensions made in HBSS. Serial fourfold dilutions from $1 / 10$ to $1 / 655360$ were then prepared for all specimens in HBSS containing a $10 \%$ suspension of a negative stool. These dilutions provided the starting material for this second series of experiments. They were coded in a similar way to the previous experiment so that neither the number of rotavirus positive stools in the experiment nor the dilution being examined was known during subsequent testing. Before testing, each sample was diluted $1 / 10$ in either Rotalex buffer (for latex agglutination) or HBSS (for the other tests). This gave final dilutions ranging from $1 / 100$ to $1 / 6553600$.

Table 3 gives the results after decoding. Similar levels of sensitivity were found with PAGE, ELISA, electron microscopy, and immunofluorescence, and no false positive results were recorded by 'any of these four tests. The immunofluorescence results àre interesting. Several of the higher dilutions of two of the positive samples (VK 1898 and VK:2516) produced a single fluorescing cell per monolayer at several increasing dilutions (Table 3 ). If the highest dilution showing a single positive cell is taken as the end point, then immunofluorescence titres were one to three dilutions higher than PAGE, ELISA, and 
Table 1 Effect of incubation time on latex agglutination (Rotalex)

\begin{tabular}{|c|c|c|c|c|c|c|c|c|c|c|c|}
\hline \multicolumn{2}{|c|}{ Specimen } & \multirow[t]{3}{*}{ Dilution factor + diluent } & \multicolumn{9}{|c|}{ Results } \\
\hline \multirow[t]{2}{*}{ Type } & \multirow[t]{2}{*}{ No } & & \multicolumn{3}{|c|}{ After 2 min } & \multicolumn{3}{|c|}{ After $10 \mathrm{~min}$} & \multicolumn{3}{|c|}{ After $20 \mathrm{~min}$} \\
\hline & & & $A$ & $B$ & $C$ & $A$ & $B$ & $C$ & $A$ & $B$ & $c$ \\
\hline $\begin{array}{l}\text { Pos } \\
\text { Pos } \\
\text { Pos } \\
\text { Pos } \\
\text { Pos } \\
\text { Pos } \\
\text { Pos } \\
\text { Pos } \\
\text { Pos }\end{array}$ & $\begin{array}{l}1 \\
2 \\
3 \\
4 \\
5 \\
6 \\
7 \\
8 \\
9\end{array}$ & $\begin{array}{l}1 / 10 \mathrm{R} \\
1 / 10 \mathrm{R} \\
1 / 10 \mathrm{R} \\
1 / 10 \mathrm{R} \\
1 / 10 \mathrm{R} \\
1 / 10 \mathrm{R} \\
1 / 10 \mathrm{R} \\
1 / 10 \mathrm{R} \\
1 / 10 \mathrm{R}\end{array}$ & $\begin{array}{l}- \\
- \\
- \\
- \\
\overline{+} \\
+ \\
+ \\
+\end{array}$ & $\begin{array}{l}- \\
- \\
+ \\
+ \\
+ \\
+ \\
+ \\
+ \\
+\end{array}$ & $\begin{array}{l}+ \\
+ \\
+ \\
\mathrm{Eq} \\
- \\
\mathrm{Eq} \\
+ \\
+ \\
+\end{array}$ & $\begin{array}{l}- \\
- \\
+ \\
- \\
- \\
- \\
+ \\
+ \\
+\end{array}$ & $\begin{array}{l}- \\
+ \\
+ \\
+ \\
+ \\
+ \\
+ \\
+ \\
+\end{array}$ & $\begin{array}{l}+ \\
+ \\
+ \\
+ \\
+ \\
+ \\
+ \\
+ \\
+\end{array}$ & $\begin{array}{l}- \\
+ \\
+ \\
+ \\
+ \\
+ \\
+ \\
+ \\
+\end{array}$ & $\begin{array}{l}- \\
+ \\
+ \\
+ \\
+ \\
+ \\
+ \\
+ \\
+\end{array}$ & $\begin{array}{l}+ \\
\text { Eq } \\
+ \\
+ \\
+ \\
+ \\
+ \\
+ \\
+\end{array}$ \\
\hline $\begin{array}{l}\text { Pos } \\
\text { Pos } \\
\text { Pos } \\
\text { Pos } \\
\text { Pos } \\
\text { Pos } \\
\text { Pos } \\
\text { Pos } \\
\text { Pos }\end{array}$ & $\begin{array}{l}1 \\
2 \\
3 \\
4 \\
5 \\
6 \\
7 \\
8 \\
9\end{array}$ & $\begin{array}{l}1 / 10 \mathrm{H} \times 1 / 10 \mathrm{R} \\
1 / 10 \mathrm{H} \times 1 / 10 \mathrm{R} \\
1 / 10 \mathrm{H} \times 1 / 10 \mathrm{R} \\
1 / 10 \mathrm{H} \times 1 / 10 \mathrm{R} \\
1 / 10 \mathrm{H} \times 1 / 10 \mathrm{R} \\
1 / 10 \mathrm{H} \times 1 / 10 \mathrm{R} \\
1 / 10 \mathrm{H} \times 1 / 10 \mathrm{R} \\
1 / 10 \mathrm{H} \times 1 / 10 \mathrm{R} \\
1 / 10 \mathrm{H} \times 1 / 10 \mathrm{R}\end{array}$ & $\begin{array}{l}+ \\
- \\
\overline{-} \\
\overline{-} \\
\overline{+} \\
\overline{-} \\
-\end{array}$ & $\begin{array}{l}+ \\
+ \\
+ \\
+ \\
+ \\
+ \\
+ \\
+ \\
+\end{array}$ & $\begin{array}{l}+ \\
+ \\
+ \\
+ \\
+ \\
+ \\
+ \\
+\end{array}$ & $\begin{array}{l}+ \\
+ \\
+ \\
+ \\
+ \\
+ \\
+ \\
+ \\
+\end{array}$ & $\begin{array}{l}+ \\
+ \\
+ \\
+ \\
+ \\
+ \\
+ \\
+ \\
+\end{array}$ & $\begin{array}{l}+ \\
+ \\
+ \\
+ \\
+ \\
+ \\
+ \\
+ \\
+\end{array}$ & $\begin{array}{l}+ \\
+ \\
+ \\
+ \\
+ \\
+ \\
+ \\
+ \\
+\end{array}$ & $\begin{array}{l}+ \\
+ \\
+ \\
+ \\
+ \\
+ \\
+ \\
+ \\
+\end{array}$ & $\begin{array}{l}+ \\
+ \\
+ \\
+ \\
+ \\
+ \\
+ \\
+ \\
+\end{array}$ \\
\hline $\begin{array}{l}\text { Neg } \\
\text { Neg } \\
\text { Neg } \\
\text { Neg } \\
\text { Neg } \\
\text { Neg } \\
\text { Neg } \\
\text { Neg } \\
\text { Neg }\end{array}$ & $\begin{array}{l}1 \\
2 \\
3 \\
4 \\
5 \\
6 \\
7 \\
8 \\
9\end{array}$ & $\begin{array}{l}1 / 10 \mathrm{R} \\
1 / 10 \mathrm{R} \\
1 / 10 \mathrm{R} \\
1 / 10 \mathrm{R} \\
1 / 10 \mathrm{R} \\
1 / 10 \mathrm{R} \\
1 / 10 \mathrm{R} \\
1 / 10 \mathrm{R} \\
1 / 10 \mathrm{R}\end{array}$ & $\begin{array}{l}\overline{-} \\
\overline{-} \\
\overline{-} \\
\overline{-} \\
\overline{-}\end{array}$ & $\begin{array}{l}\overline{-} \\
\overline{-} \\
\overline{-} \\
\overline{-} \\
\text { Eq } \\
\mathrm{Eq} \\
\mathrm{Eq}\end{array}$ & $\begin{array}{l}- \\
\overline{-} \\
- \\
- \\
- \\
\overline{+} \\
+\end{array}$ & $\begin{array}{l}- \\
\overline{-} \\
\overline{-} \\
\overline{-} \\
\overline{-} \\
-\end{array}$ & $\begin{array}{l}\overline{-} \\
\overline{-} \\
\overline{-} \\
\overline{E q} \\
+ \\
\text { Eq } \\
\text { Eq }\end{array}$ & $\begin{array}{l}- \\
+ \\
- \\
+ \\
- \\
- \\
+ \\
+\end{array}$ & $\begin{array}{l}- \\
\overline{-} \\
+ \\
\overline{+} \\
+ \\
+ \\
\overline{+}\end{array}$ & $\begin{array}{l}- \\
\overline{-} \\
\bar{R} \\
\overline{+} \\
+ \\
+ \\
\mathbf{R} \\
+\end{array}$ & $\begin{array}{l}- \\
+ \\
- \\
+ \\
\text { Eq } \\
\text { Eq } \\
\text { Eq } \\
+ \\
+\end{array}$ \\
\hline $\begin{array}{l}\text { Neg } \\
\text { Neg } \\
\text { Neg } \\
\text { Neg } \\
\text { Neg } \\
\text { Neg } \\
\text { Neg } \\
\text { Neg } \\
\text { Neg }\end{array}$ & $\begin{array}{l}1 \\
2 \\
3 \\
4 \\
5 \\
6 \\
7 \\
8 \\
9\end{array}$ & $\begin{array}{l}1 / 10 \mathrm{H} \times 1 / 10 \mathrm{R} \\
1 / 10 \mathrm{H} \times 1 / 10 \mathrm{R} \\
1 / 10 \mathrm{H} \times 1 / 10 \mathrm{R} \\
1 / 10 \mathrm{H} \times 1 / 10 \mathrm{R} \\
1 / 10 \mathrm{H} \times 1 / 10 \mathrm{R} \\
1 / 10 \mathrm{H} \times 1 / 10 \mathrm{R} \\
1 / 10 \mathrm{H} \times 1 / 10 \mathrm{R} \\
1 / 10 \mathrm{H} \times 1 / 10 \mathrm{R} \\
1 / 10 \mathrm{H} \times 1 / 10 \mathrm{R}\end{array}$ & $\begin{array}{l}- \\
\overline{-} \\
\overline{-} \\
\overline{-} \\
- \\
-\end{array}$ & $\begin{array}{l}- \\
- \\
\text { Eq } \\
- \\
- \\
- \\
- \\
-\end{array}$ & $\begin{array}{l}- \\
\overline{+} \\
- \\
- \\
- \\
- \\
- \\
-\end{array}$ & $\begin{array}{l}- \\
\overline{-} \\
\overline{-} \\
\overline{-} \\
\overline{-} \\
-\end{array}$ & $\begin{array}{l}- \\
- \\
\text { Eq } \\
- \\
- \\
- \\
- \\
-\end{array}$ & $\begin{array}{l}\text { Eq } \\
- \\
- \\
\overline{+} \\
- \\
- \\
-\end{array}$ & $\begin{array}{l}- \\
\overline{-} \\
\overline{-} \\
\overline{-} \\
\overline{-} \\
\overline{-}\end{array}$ & $\begin{array}{l}- \\
\bar{R} \\
- \\
- \\
- \\
- \\
- \\
-\end{array}$ & $\begin{array}{l}\text { Eq } \\
- \\
- \\
- \\
+ \\
\overline{E q} \\
\overline{-}\end{array}$ \\
\hline
\end{tabular}

Eighteen stool samples (nine previously positive for rotavirus by electron microscopy) were examined at dilutions of $1 / 10$ ( $1 / 10 \mathrm{R})$ and $1 / 100(1 / 10 \mathrm{H} \times 1 / 10 \mathrm{R})$.

$1 / 10 \mathrm{R}=$ sample diluted in $1 / 10$ in Rotalex buffer.

$1 / 10 \mathrm{H} \times 1 / 10 \mathrm{R}=$ sample diluted $1 / 10$ in Hank's balanced salt solution and then a further $1 / 10$ in Rotalex buffer.

Pos = positive by electron microscopy; neg = negative by electron microscopy

$+=$ agglutination visible using hand lens.

$-=$ no agglutination visible using hand lens.

$\mathrm{Eq}=$ equivocal.

$\mathbf{R}=$ to be repeated (subsequently found to be negative).

A, B, C = different observers.

$\mathrm{A}=$ most previous experience, $\mathrm{B}=$ some previous experience, $\mathrm{C}=$ no previous experience.

All specimens were read individually under code.

electron microscopy, although this depended on finding the single cell.

The lowest titres were obtained with Rotalex and no false positive results were recorded by the most experienced reader (A) of this test (Table 3). Even though higher titres were recorded for the positive samples by the less experienced readers B and C, they also scored false positive results with dilutions of the negative samples.

In this experiment, the importance of experience in reading the Rotalex test became apparent. The most experienced reader, $A$, recorded the lowest titres but no false positive results. The less experi- 
Table 2 Detection of rotavirus in clinical specimens using Rotalex, enzyme linked immunosorbent assay (ELISA), electron microscopy $(E M)$, polyacrylamide gel electrophoresis (PAGE), and immunoftuorescence (IF)

\begin{tabular}{|c|c|c|c|c|c|c|c|c|c|c|}
\hline \multicolumn{2}{|c|}{ Specimen } & \multicolumn{4}{|l|}{ Rotalex } & \multirow{3}{*}{$\begin{array}{l}\text { Dilution } \\
\text { factor }+ \\
\text { diluent }\end{array}$} & \multirow{3}{*}{$\begin{array}{l}\text { ELISA } \\
\text { result } O D\end{array}$} & \multirow{3}{*}{$\begin{array}{l}\text { EM } \\
\text { result }\end{array}$} & \multirow{3}{*}{$\begin{array}{l}\text { PAGE } \\
\text { result }\end{array}$} & \multirow{3}{*}{$\begin{array}{l}\text { IF } \\
\text { result }\end{array}$} \\
\hline \multirow[t]{2}{*}{ Type } & \multirow[t]{2}{*}{ No } & \multirow[t]{2}{*}{ Dilution factor + diluent } & \multicolumn{3}{|c|}{ Result } & & & & & \\
\hline & & & $\boldsymbol{A}$ & $\boldsymbol{B}$ & $C$ & & & & & \\
\hline $\begin{array}{l}\text { Pos } \\
\text { Pos } \\
\text { Pos } \\
\text { Pos } \\
\text { Pos } \\
\text { Pos } \\
\text { Pos } \\
\text { Pos } \\
\text { Pos }\end{array}$ & $\begin{array}{l}1 \\
2 \\
3 \\
4 \\
5 \\
6 \\
7 \\
8 \\
9\end{array}$ & $\begin{array}{l}1 / 10 \mathbf{R} \\
1 / 10 \mathbf{R} \\
1 / 10 \mathbf{R} \\
1 / 10 \mathbf{R} \\
1 / 10 \mathbf{R} \\
1 / 10 \mathbf{R} \\
1 / 10 \mathbf{R} \\
1 / 10 \mathbf{R} \\
1 / 10 \mathbf{R}\end{array}$ & $\begin{array}{l}- \\
+ \\
+ \\
+ \\
- \\
+ \\
+ \\
+ \\
+\end{array}$ & $\begin{array}{l}- \\
+ \\
+ \\
+ \\
- \\
+ \\
+ \\
+ \\
+\end{array}$ & $\begin{array}{l}+ \\
\text { Eq } \\
+ \\
+ \\
+ \\
+ \\
+ \\
+ \\
+\end{array}$ & $\begin{array}{l}1 / 10 \mathrm{H} \\
1 / 10 \mathrm{H} \\
1 / 10 \mathrm{H} \\
1 / 10 \mathrm{H} \\
1 / 10 \mathrm{H} \\
1 / 10 \mathrm{H} \\
1 / 10 \mathrm{H} \\
1 / 10 \mathrm{H} \\
1 / 10 \mathrm{H}\end{array}$ & $\begin{array}{l}+ \\
+ \\
+ \\
+ \\
+ \\
+ \\
+ \\
+ \\
+\end{array}$ & $\begin{array}{l}++++ \\
+++ \\
+++ \\
+++ \\
++ \\
+++ \\
+++ \\
+++ \\
+++\end{array}$ & $\begin{array}{l}+ \\
+ \\
+ \\
+ \\
+ \\
+ \\
+ \\
+ \\
+\end{array}$ & $\begin{array}{l}+ \\
+ \\
+ \\
+ \\
+ \\
+ \\
+ \\
+ \\
+\end{array}$ \\
\hline $\begin{array}{l}\text { Pos } \\
\text { Pos } \\
\text { Pos } \\
\text { Pos } \\
\text { Pos } \\
\text { Pos } \\
\text { Pos } \\
\text { Pos } \\
\text { Pos }\end{array}$ & $\begin{array}{l}1 \\
2 \\
3 \\
4 \\
5 \\
6 \\
7 \\
8 \\
9\end{array}$ & $\begin{array}{l}1 / 10 \mathrm{H} \times 1 / 10 \mathrm{R} \\
1 / 10 \mathrm{H} \times 1 / 10 \mathrm{R} \\
1 / 10 \mathrm{H} \times 1 / 10 \mathrm{R} \\
1 / 10 \mathrm{H} \times 1 / 10 \mathrm{R} \\
1 / 10 \mathrm{H} \times 1 / 10 \mathrm{R} \\
1 / 10 \mathrm{H} \times 1 / 10 \mathrm{R} \\
1 / 10 \mathrm{H} \times 1 / 10 \mathrm{R} \\
1 / 10 \mathrm{H} \times 1 / 10 \mathrm{R} \\
1 / 10 \mathrm{H} \times 1 / 10 \mathrm{R}\end{array}$ & $\begin{array}{l}+ \\
+ \\
+ \\
+ \\
+ \\
+ \\
+ \\
+ \\
+\end{array}$ & $\begin{array}{l}+ \\
+ \\
+ \\
+ \\
+ \\
+ \\
+ \\
+ \\
+\end{array}$ & $\begin{array}{l}+ \\
+ \\
+ \\
+ \\
+ \\
+ \\
+ \\
+ \\
+\end{array}$ & $\begin{array}{l}1 / 100 \mathrm{H} \\
1 / 100 \mathrm{H} \\
1 / 100 \mathrm{H} \\
1 / 100 \mathrm{H} \\
1 / 100 \mathrm{H} \\
1 / 100 \mathrm{H} \\
1 / 100 \mathrm{H} \\
1 / 100 \mathrm{H} \\
1 / 100 \mathrm{H}\end{array}$ & $\begin{array}{l}+ \\
+ \\
+ \\
+ \\
+ \\
+ \\
+ \\
+ \\
+\end{array}$ & $\begin{array}{l}+++ \\
+++ \\
++ \\
\text { occ } \\
+ \\
+++ \\
++ \\
++ \\
++\end{array}$ & $\begin{array}{l}+ \\
+ \\
+ \\
+ \\
+ \\
+ \\
+ \\
+ \\
+\end{array}$ & $\begin{array}{l}+ \\
+ \\
+ \\
+ \\
+ \\
+ \\
+ \\
+ \\
+\end{array}$ \\
\hline $\begin{array}{l}\text { Neg } \\
\text { Neg } \\
\text { Neg } \\
\text { Neg } \\
\text { Neg } \\
\text { Neg } \\
\text { Neg } \\
\text { Neg } \\
\text { Neg }\end{array}$ & $\begin{array}{l}1 \\
2 \\
3 \\
4 \\
5 \\
6 \\
7 \\
8 \\
9\end{array}$ & $\begin{array}{l}1 / 10 \mathrm{R} \\
1 / 10 \mathrm{R} \\
1 / 10 \mathrm{R} \\
1 / 10 \mathrm{R} \\
1 / 10 \mathrm{H} \\
1 / 10 \mathrm{R} \\
1 / 10 \mathrm{R} \\
1 / 10 \mathrm{R} \\
1 / 10 \mathrm{R}\end{array}$ & $\begin{array}{l}- \\
- \\
\overline{+} \\
\overline{+} \\
+ \\
\overline{+} \\
+\end{array}$ & $\begin{array}{l}- \\
- \\
- \\
\overline{+} \\
+ \\
\overline{+}\end{array}$ & $\begin{array}{l}- \\
+ \\
- \\
+ \\
\mathrm{Eq} \\
\mathrm{Eq} \\
\mathrm{Eq} \\
+ \\
+\end{array}$ & $\begin{array}{l}1 / 10 \mathrm{H} \\
1 / 10 \mathrm{H} \\
1 / 10 \mathrm{H} \\
1 / 10 \mathrm{H} \\
1 / 10 \mathrm{H} \\
1 / 10 \mathrm{H} \\
1 / 10 \mathrm{H} \\
1 / 10 \mathrm{H} \\
1 / 10 \mathrm{H}\end{array}$ & $\begin{array}{l}- \\
- \\
- \\
- \\
- \\
- \\
-\end{array}$ & $\begin{array}{l}- \\
- \\
- \\
- \\
- \\
- \\
- \\
-\end{array}$ & $\begin{array}{l}- \\
- \\
- \\
- \\
- \\
- \\
-\end{array}$ & $\begin{array}{l}- \\
- \\
- \\
- \\
- \\
- \\
-\end{array}$ \\
\hline $\begin{array}{l}\text { Neg } \\
\text { Neg } \\
\text { Neg } \\
\text { Neg } \\
\text { Neg } \\
\text { Neg } \\
\text { Neg } \\
\text { Neg } \\
\text { Neg }\end{array}$ & $\begin{array}{l}1 \\
2 \\
3 \\
4 \\
5 \\
6 \\
7 \\
8 \\
9\end{array}$ & $\begin{array}{l}1 / 10 \mathrm{H} \times 1 / 10 \mathrm{R} \\
1 / 10 \mathrm{H} \times 1 / 10 \mathrm{R} \\
1 / 10 \mathrm{H} \times 1 / 10 \mathrm{R} \\
1 / 10 \mathrm{H} \times 1 / 10 \mathrm{R} \\
1 / 10 \mathrm{H} \times 1 / 10 \mathrm{R} \\
1 / 10 \mathrm{H} \times 1 / 10 \mathrm{R} \\
1 / 10 \mathrm{H} \times 1 / 10 \mathrm{R} \\
1 / 10 \mathrm{H} \times 1 / 10 \mathrm{R} \\
1 / 10 \mathrm{H} \times 1 / 10 \mathrm{R}\end{array}$ & $\begin{array}{l}- \\
- \\
- \\
- \\
- \\
- \\
-\end{array}$ & $\begin{array}{l}- \\
- \\
- \\
- \\
- \\
- \\
-\end{array}$ & $\begin{array}{l}\mathrm{Eq} \\
\overline{-} \\
\overline{-} \\
\overline{+} \\
\overline{\mathrm{Eq}} \\
\overline{-}\end{array}$ & $\begin{array}{l}1 / 100 \mathrm{H} \\
1 / 100 \mathrm{H} \\
1 / 100 \mathrm{H} \\
1 / 100 \mathrm{H} \\
1 / 100 \mathrm{H} \\
1 / 100 \mathrm{H} \\
1 / 100 \mathrm{H} \\
1 / 100 \mathrm{H} \\
1 / 100 \mathrm{H}\end{array}$ & $\begin{array}{l}- \\
- \\
- \\
- \\
- \\
- \\
-\end{array}$ & $\begin{array}{l}- \\
- \\
- \\
- \\
- \\
- \\
-\end{array}$ & $\begin{array}{l}- \\
- \\
- \\
- \\
- \\
- \\
-\end{array}$ & $\begin{array}{l}- \\
- \\
- \\
- \\
- \\
- \\
-\end{array}$ \\
\hline
\end{tabular}

Eighteen stool samples (nine previously positive for rotavirus by electron microscopy) were examined at dilutions of $1 / 10$ (1/10 $R$ or $1 / 10 \mathrm{H})$ and $1 / 100(1 / 10 \mathrm{H} \times 1 / 10 \mathrm{R}$ or $1 / 100 \mathrm{H})$.

$1 / 10 \mathrm{R}=$ sample diluted $1 / 10$ in Rotalex buffer.

$1 / 10 \mathrm{H}=$ sample diluted $1 / 10$ in Hank's balanced salt solution.

$1 / 10 \mathrm{H} \times 1 / 10 \mathrm{R}=1 / 10 \mathrm{H}$ diluted a further $1 / 10$ in Rotalex buffer.

$1 / 100 \mathrm{H}=$ sample diluted $1 / 100$ in Hank's balanced salt solution.

Pos = positive by electron microscopy; neg = negative by electron microscopy.

$\left.\begin{array}{l}\text { Rotalex } t=\text { agglutination visible using hand lens } \\ \text { Rotalex }-=\text { no agglutination using hand lens }\end{array}\right\}$ results read at $20 \mathrm{~min}$.

Rotalex $\mathrm{Eq}=$ equivocal.

$A, B, C=$ different observers.

$A=$ most previous experience, $B=$ some previous experience, $C=$ no previous experience.

ELISA: specimens scored as positive by having an $O D$ of $>2 x$ negative control.

Electron microscopy: $++++>100$ virus particles/grid square (v/gs). $+++20-100 \mathrm{v} / g s ;++5-20 \mathrm{v} / g s ;+1-5 \mathrm{v} / \mathrm{gs} ;$ occ $<1 \mathrm{v} / \mathrm{gs}$.

All specimens were read individually under code.

tal glass surface and is based on the agglutination of latex particles, which can be read with the naked eye or a hand lens. But we encountered sensitivity problems and lack of specificity when the instructions in the Rotalex kit were strictly adhered to. We found that reading the test after 2 min, as suggested by the manufacturer, resulted in the detection of fewer positive results and that extending the reaction time to $20 \mathrm{~min}$ increased the chances of detecting rotavirus (Table 1).

Preparation of the $10 \%$ stool suspensions directly in Rotalex buffer, again as recommended by the manufacturer, resulted in the recording of false positive results, which increased with time of incubation (Table 1). We found, however, that preparation of the initial $10 \%$ stool extracts in HBSS and subsequently diluting $1 / 10$ in Rotalex buffer removed this non-specificity. The increase in specificity may be due to changes in $\mathrm{pH}$ or ionic effects in the presence of HBSS.

Experience in reading latex agglutination tests appears to be essential since discrepancies were 
Table 3 Sensitivity of Rotalex for detecting rotavirus in comparison with enzyme linked immunosorbent assay (ELISA), electron microscopy (EM), polyacrylamide gel electrophoresis (PAGE), and immunoftuorescence (IF)

\begin{tabular}{|c|c|c|c|c|c|c|c|c|c|}
\hline \multicolumn{2}{|c|}{ Specimen } & \multirow[t]{2}{*}{ Dilution factor } & \multicolumn{3}{|c|}{ Rotalex result } & \multirow{2}{*}{$\begin{array}{l}\text { ELISA } \\
\text { result }\end{array}$} & \multirow{2}{*}{$\begin{array}{l}E M \\
\text { result }\end{array}$} & \multirow{2}{*}{$\begin{array}{l}\text { PAGE } \\
\text { result }\end{array}$} & \multirow{2}{*}{$\begin{array}{l}\text { IF } \\
\text { result }\end{array}$} \\
\hline Code & Type & & $A$ & $B$ & $C$ & & & & \\
\hline I & $\begin{array}{l}\text { ROTA } \\
++\end{array}$ & $\begin{array}{r}100 \\
400 \\
1600 \\
6400 \\
25600 \\
102400 \\
409600 \\
1638400 \\
6553600\end{array}$ & $\begin{array}{l}+ \\
+ \\
+ \\
- \\
- \\
- \\
- \\
-\end{array}$ & $\begin{array}{l}+ \\
+ \\
+ \\
- \\
- \\
- \\
- \\
-\end{array}$ & $\begin{array}{l}+ \\
+ \\
+ \\
+ \\
+ \\
+ \\
- \\
-\end{array}$ & $\begin{array}{l}+ \\
+ \\
+ \\
+ \\
+ \\
+ \\
- \\
-\end{array}$ & $\begin{array}{l}+++ \\
+++ \\
+ \\
+ \\
+ \\
+ \\
- \\
-\end{array}$ & $\begin{array}{l}+ \\
+ \\
+ \\
+ \\
+ \\
- \\
- \\
-\end{array}$ & $\begin{array}{l}++++ \\
+++ \\
+++ \\
++ \\
++ \\
++ \\
+ \\
+ \\
+\end{array}$ \\
\hline II & $\begin{array}{l}\text { VK } \\
1898 \\
\text { ROTA } \\
++\end{array}$ & $\begin{array}{r}100 \\
400 \\
1600 \\
6400 \\
25600 \\
102400 \\
409600 \\
1638400 \\
6553600\end{array}$ & $\begin{array}{l}+ \\
+ \\
+ \\
- \\
- \\
- \\
- \\
-\end{array}$ & $\begin{array}{l}+ \\
+ \\
+ \\
+ \\
- \\
- \\
- \\
- \\
-\end{array}$ & $\begin{array}{l}+ \\
+ \\
+ \\
+ \\
\overline{E q} \\
= \\
-\end{array}$ & $\begin{array}{l}+ \\
+ \\
+ \\
+ \\
+ \\
- \\
- \\
-\end{array}$ & $\begin{array}{l}+++ \\
+++ \\
+++ \\
++ \\
\text { occ } \\
- \\
- \\
- \\
-\end{array}$ & $\begin{array}{l}+ \\
+ \\
+ \\
+ \\
- \\
- \\
- \\
-\end{array}$ & $\begin{array}{l}++++ \\
++++ \\
+++ \\
++ \\
++ \\
+ \\
+ \\
+ \\
+\end{array}$ \\
\hline III & $\begin{array}{l}\text { VK } \\
2516 \\
\text { ROTA } \\
+++\end{array}$ & $\begin{array}{r}100 \\
400 \\
1600 \\
\\
6400 \\
25600 \\
102400 \\
409600 \\
1638400 \\
6553600\end{array}$ & $\begin{array}{l}+ \\
+ \\
+ \\
- \\
- \\
- \\
- \\
-\end{array}$ & $\begin{array}{l}+ \\
+ \\
+ \\
+ \\
+ \\
+ \\
- \\
-\end{array}$ & $\begin{array}{l}+ \\
+ \\
+ \\
- \\
- \\
- \\
- \\
-\end{array}$ & $\begin{array}{l}+ \\
- \\
+ \\
+ \\
- \\
- \\
- \\
-\end{array}$ & $\begin{array}{l}+ \\
+ \\
- \\
+ \\
+ \\
- \\
- \\
-\end{array}$ & $\begin{array}{l}+ \\
+ \\
+ \\
+ \\
+ \\
- \\
- \\
-\end{array}$ & $\begin{array}{l}++++ \\
++ \\
++ \\
+ \\
+ \\
+ \\
+ \\
+ \\
-\end{array}$ \\
\hline IV & $\begin{array}{l}\text { VK } \\
2488\end{array}$ & $\begin{array}{r}100 \\
400 \\
\\
\\
1600 \\
6400 \\
25600 \\
102400 \\
409600 \\
1638400 \\
6553600 \\
\end{array}$ & $\begin{array}{l}- \\
\overline{-} \\
\overline{-} \\
- \\
\overline{-} \\
\overline{-} \\
-\end{array}$ & $\begin{array}{l}- \\
\overline{+} \\
- \\
- \\
- \\
+ \\
- \\
-\end{array}$ & $\begin{array}{l}\overline{+} \\
+ \\
+ \\
+ \\
\overline{E q} \\
\overline{-} \\
\bar{E}\end{array}$ & $\begin{array}{l}- \\
\overline{-} \\
- \\
- \\
- \\
- \\
- \\
-\end{array}$ & $\begin{array}{l}- \\
\overline{-} \\
\overline{-} \\
\overline{-} \\
\overline{-} \\
\overline{-}\end{array}$ & $\begin{array}{l}- \\
\overline{-} \\
\overline{-} \\
\overline{-} \\
\overline{-} \\
\overline{-} \\
-\end{array}$ & $\begin{array}{l}- \\
\overline{-} \\
- \\
- \\
- \\
- \\
- \\
-\end{array}$ \\
\hline
\end{tabular}

Serial fourfold dilutions of four coded faecal specimens (I, II, III, IV) were made and dilutions were examined as individual samples under code by the five methods.

NVS = no viruses seen by electron microscopy.

Rotalex $+=$ agglutination visible using hand lens.

Rotalex $-=$ no agglutination using hand lens.

Rotalex $\mathrm{Eq}=$ equivocal.

ELISA: specimens scored as positive by having an OD of $>2 x$ negative control.

Electron microscopy: $++++>100$ virus particles/grid square (v/gs); $+++20-100 \mathrm{v} / \mathrm{gs} ;++5-20 \mathrm{v} / \mathrm{gs} ;+1-5 \mathrm{v} / \mathrm{gs} ;$ occ $<1 \mathrm{v} / \mathrm{gs}$.

Immunofluorescence: $++++>100$ fluorescing cells/monolayer $(\mathrm{fc} / \mathrm{m}) ;+++51-100 \mathrm{fc} / \mathrm{m} ;++2-50 \mathrm{fc} / \mathrm{m} ;+1 \mathrm{fc} / \mathrm{m}$.

A, B, C = different observers.

$A=$ most previous experience; $B=$ some previous experience; $C=$ no previous experience.

All specimens were read individually under code.

noted in the reading of the results among the three observers. Observer A, who had read the results of all preliminary experiments undertaken with Rotalex and who was therefore the most experienced, did not record false positive results with any of the specimens diluted first in HBSS and obtained complete agreement with the other tests when reading Rotalex after $20 \mathrm{~min}$. These results depended on the observer's decision to record the results as definitely positive or definitely negative. Some could otherwise have been scored as doubtful. Observer C was the least experienced and recorded more false positive results than observer $B$, who was of intermediate experience, although both correctly read all known positive results diluted $1 / 100$ after $20 \mathrm{~min}$ (Table 2).

When used according to the manufacturer's instructions for the detection of rotavirus the Rotalex kit shows a lack of both specificity and sensitivity (Tables 1 and 2). These findings are in general agreement with those of other workers using latex agglutination. ${ }^{1814}$ All specimens tested had been stored beforehand. We believe that the same results would have been obtained with fresh specimens.

The present investigation showed, however, that when carried out in the presence of HBSS and read after $20 \mathrm{~min}$ by an experienced observer Rotalex 
can give results which correlate well with those of other techniques such as electron microscopy, ELISA, PAGE, and immunofluorescence (Tables 2 and 3). The latter techniques, when applied to detected rotavirus, correlated with each other to the same degree, although immunofluorescence surprisingly showed substantially higher end points when a single fluorescing cell per monolayer was accepted as indicating positivity (Table 3 ). We attribute this finding to the fact that a single infectious virus particle may not necessarily register in the other tests but may initiate the production of antigens in a cell which can be detected by immunofluorescence.

Our experience suggests that Rotalex requires four basic modifications before it can be considered as a possible standard method for detecting rotavirus:

1 Dilution: in our hands the Rotalex buffer is not suitable, allowing too many false positive results.

2 Incubation: $2 \mathrm{~min}$ is too short. Twenty minutes gave better results but added considerably to the time required to test a large number of stools.

3 Experience: performance improved substantially with experience.

4 Test base: we found the standard drops too large for a $25 \mathrm{~mm} \times 75 \mathrm{~mm}$ microscope slide as the results were best assessed on a flowing drop. A hand lens was thought by all observers to be essential. Plastic surfaces are unsuitable.

A modified test which can be done on $50 \mathrm{~mm}$ square glass plates with commercially available reagents may find useful application in developing countries where there is a general lack of high technology. Like ELISA and immunofluorescence, however, it is an antibody based test and would not detect the antigenically atypical rotaviruses which have been reported..$^{21}$ These can be detected only by electron microscopy and PAGE and, so far, are distinguishable by PAGE alone.

This work was supported by a grant from the Medical Research Council. The initial Rotalex kits were a gift from Dr Alan Volkers, Northumbria Biologicals. The authors gratefully acknowledge Dr M Helmy, Miss J McQuillin, Miss J Norden, Dr R Scott, and Mr M Younghusband for their excellent assistance in these studies and Miss Y Caruana for typing the manuscript.

\section{References}

' Banatvala JE, Totterdell B, Chrystie IL, Woode GN. In vitro detection of human rotaviruses. Lancet 1975 ;ii:821.

${ }^{2}$ Moosai RB, Gardner PS, Almeida JD, Greenaway MA. A simple immunofluorescent technique for the detection of human rotavirus. J Med Virol 1979;3:189-94.

${ }^{3}$ Yolken RH, Wyatt RG, Zissis G, et al. Epidemiology of human rotavirus types 1 and 2 as studied by enzyme-linked immunosorbent assay. $N$ Engl J Med 1978;299:1156-61.

${ }^{4}$ Grauballe PC, Vestergaard BF, Meyling A, Genner J. Optimized enzyme-linked immunosorbent assay for detection of human and bovine rotavirus in stools: comparison with electron microscopy, immunoelectro-osmophoresis and fluorescent antibody techniques. $J$ Med Virol 1981;7:29-40.

${ }^{5}$ Kalica AR, Purcell RH, Sereno MM, et al. A microtiter solidphase radioimmunoassay for detection of the human reoviruslike agent in stools. J Immunol 1977; 118:1275-9.

- Harris CC, Yolken RH, Kroken H, Hsu IC. Ultrasensitive enzymatic radioimmunoassay. Application to detection of cholera toxin and rotavirus. Proc Natl Acad Sci USA 1979; 76:5336-39.

' Grauballe PC, Genner J, Meyling A, Hornsleth A. Rapid diagnosis of rotavirus infections: comparison of electron microscopy and immunoelectro-osmophoresis for the detection of rotavirus in human infantile gastroenteritis. J Gen Virol 1977;35:203-18.

${ }^{8}$ Middleton PJ, Petric M, Hewitt CM, Szymanski MT, Tam JS. Counter-immuno-electro-osmophoresis for the detection of infantile gastroenteritis virus (orbi-group) antigen and antibody. J Clin Pathol 1976;29:191-7.

" Flewett TH, Davies H, Bryden AS, Robertson MJ. Diagnostic electron microscopy of faeces. II Acute gastroenteritis associated with reovirus-like particles. J Clin Pathol 1974;27:60814.

${ }^{10}$ Madeley CR, Cosgrove BP, Bell EJ, Fallon RJ. Stool viruses in babies in Glasgow. 1. Hospital admissions with diarrhoea. $J$ Hyg (Camb) 1977;78:261-73.

" Espejo RT, Munoz O, Serafin F, Romero P. Shift in the prevalent human rotavirus detected by ribonucleic acid segment differences. Infect Immun 1980;27:351-4.

12 Herring AJ, Inglis NF, Ojeh CK, Snodgrass DR, Menzies JD. Rapid diagnosis of rotavirus infection by direct detection of viral nucleic acid in silver-stained polyacrylamide gels. $J$ Clin Microbiol 1982;16:473-7.

${ }^{13}$ Moosai RB, Alcock R, Madeley CR. A cryptogram for recording rotavirus strains: the Rotacode. J Hyg (Camb) 1984;93:23749.

${ }^{14}$ Gardner PS, McQuillin J. Rapid virus diagnosis: application of immunofuorescence, 2nd ed. London: Butterworth. 1980.

is Laemmli UK. Cleavage of structural proteins during the assembly of the head of bacteriophage T4. Nature 1970;227:680-5.

${ }^{16}$ Beards GM, Campbell AD, Cottrell NR, et al. Enzyme-linked immunosorbent assays based on polyclonal and monoclonal antibodies for rotavirus detection. J Clin Microbiol 1984; 19:248-54.

${ }^{17}$ Peiris JSM, Porterfield JS, Roehrig T. Monoclonal antibodies against the flavivirus West Nile. J Gen Virol 1982;58:283-9.

${ }^{1 *}$ Haikala OJ, Kolkonen JC, Leinonen MK. Nurmi T, Mäntyjärvi R, Sarkkinen HR. Rapid detection of rotavirus in stools by latex agglutination: Comparison with radioimmunoassay and electron microscopy and clinical evaluation of the test. J Med Virol 1983;11:91-7.

${ }^{14}$ Cevenini R, Rumpaniesi F, Mazzarachio R, Donati M, Falcieri E, Lazzari R. Evaluation of a new latex agglutination test for detecting human rotavirus in faeces. Journal of Infection 1983; 7:130-33.

${ }^{20}$ Snodgrass DR, Herring AJ, Campbell I, Inglis JM, Hargreaves FD. Comparison of atypical rotaviruses from calves, piglets, lambs and man. J Gen Virol 1984;65:909-14.

${ }^{21}$ Hung Tao, Chen Guangmu, Wang Changan, et al. Waterborne outbreak of rotavirus diarrhoea in adults in China caused by a novel rotavirus. Lancet 1984;i:1139-42.

Requests for reprints to: Professor CR Madeley, Department of Virology, The Royal Victoria Infirmary, Queen Victoria Road, Newcastle upon Tyne NE1 4LP, England. 